\title{
SHORT WALK ADVENTURES
}

\author{
ARMIN STRAUB AND WADIM ZUDILIN
}

To the memory of Jon Borwein, who convinced us that a short walk can be adventurous

\begin{abstract}
We review recent development of short uniform random walks, with a focus on its connection to (zeta) Mahler measures and modular parametrisation of the density functions. Furthermore, we extend available "probabilistic" techniques to cover a variation of random walks and reduce some three-variable Mahler measures, which are conjectured to evaluate in terms of $L$-values of modular forms, to hypergeometric form.
\end{abstract}

\section{INTRODUCTION}

At some stages of our careers we were approached by Jon Borwein to collaborate on a theme that sounded rather off topic to us, who had interests in number theory, combinatorics and related special functions. Somewhat unexpectedly, the theme has become a remarkable research project with several outcomes (including [9, 10, 11, to list a few), a project which we continue to enjoy after the sudden loss of Jon... This note serves as a summary to our recent discoveries that certain "probabilistic" techniques apply usefully to tackling difficult problems on the border of analysis, number theory and differential equations; in particular, in evaluating multi-variable Mahler measures. Our principal novelties are given in Theorems 13, these include hypergeometric reduction of the Mahler measures of the three-variable polynomials

$$
1+x_{1}+x_{2}+x_{3}+x_{2} x_{3} \text { and }\left(1+x_{1}\right)^{2}+x_{2}+x_{3},
$$

as well as the (hypergeometric) factorisation of a related differential operator for the Apéry-like sequence

$$
\sum_{k=0}^{n}\left(\begin{array}{l}
n \\
k
\end{array}\right)^{2}\left(\begin{array}{c}
2 k \\
k
\end{array}\right)^{2}, \quad \text { where } n=0,1,2, \ldots
$$

Echoing Jon's "a short walk can be beautiful" [8, we add that "a short walk can be adventurous."

Date: 18 January 2018. Revised: 29 April 2018.

2010 Mathematics Subject Classification. Primary 11Y60; Secondary 11F03, 11G55, 33C20, 33E05, 33E30, 44A15, 60G50.

Key words and phrases. Uniform random walk; Mahler measure; modular function; modular form; $L$-value; arithmetic differential equation; hypergeometric function.

Both authors acknowledge support of the Max Planck Society during their stays at the Max Planck Institute for Mathematics (Bonn, Germany) in the years 2015-2017. The second author is partially supported by Laboratory of Mirror Symmetry NRU HSE, RF government grant, ag. no. 14.641.31.0001. 


\section{UNIFORM RANDOM WALKS}

An $N$-step uniform random walk is a planar walk that starts at the origin and consists of $N$ steps of length 1 each taken into a uniformly random direction. Let $X_{N}$ be the distance to the origin after these $N$ steps. The $s$-th moments $W_{N}(s)$ of $X_{N}$ can be computed [11] via the formula

$$
\begin{aligned}
W_{N}(s) & =\int \cdots \int_{[0,1]^{N}}\left|e^{2 \pi i \theta_{1}}+\cdots+e^{2 \pi i \theta_{N}}\right|^{s} \mathrm{~d} \theta_{1} \cdots \mathrm{d} \theta_{N} \\
& =\int \cdots \int_{[0,1]^{N-1}}\left|1+e^{2 \pi i \theta_{1}}+\cdots+e^{2 \pi i \theta_{N-1}}\right|^{s} \mathrm{~d} \theta_{1} \cdots \mathrm{d} \theta_{N-1},
\end{aligned}
$$

and are related to the (probability) density function $p_{N}(x)$ of $X_{N}$ via

$$
W_{N}(s)=\int_{0}^{\infty} x^{s} p_{N}(x) \mathrm{d} x=\int_{0}^{N} x^{s} p_{N}(x) \mathrm{d} x .
$$

That is, $p_{N}(x)$ can then be obtained as the inverse Mellin transform of $W_{N}(s-1)$. Finally, note that the even moments $W_{3}(2 n)$ and $W_{4}(2 n)$ (which are, clearly, positive integers) can be identified with the odd moments of $I_{0}(t) K_{0}(t)^{2}$ and $I_{0}(t) K_{0}(t)^{3}$, respectively, where $I_{0}(t)$ and $K_{0}(t)$ denote the modified Bessel functions of the first and second kind. Namely, for $n=1,2, \ldots$ we have [6]

$$
W_{3}(2 n)=\frac{3^{2 n+3 / 2}}{\pi 2^{2 n} n !^{2}} \int_{0}^{\infty} t^{2 n+1} I_{0}(t) K_{0}(t)^{2} \mathrm{~d} t
$$

and

$$
W_{4}(2 n)=\frac{4^{2 n+2}}{\pi^{2} n !^{2}} \int_{0}^{\infty} t^{2 n+1} I_{0}(t) K_{0}(t)^{3} \mathrm{~d} t
$$

\section{Zeta Mahler MEASURES}

For a non-zero Laurent polynomial $P\left(x_{1}, \ldots, x_{N}\right) \in \mathbb{C}\left[x_{1}^{ \pm 1}, \ldots, x_{N}^{ \pm 1}\right]$, its zeta Mahler measure [3] is defined by

$$
Z(P ; s)=\int \cdots \int_{[0,1]^{N}}\left|P\left(e^{2 \pi i \theta_{1}}, \ldots, e^{2 \pi i \theta_{N}}\right)\right|^{s} \mathrm{~d} \theta_{1} \cdots \mathrm{d} \theta_{N},
$$

and its logarithmic Mahler measure is

$$
\mathrm{m}(P)=\left.\frac{\mathrm{d} Z(P ; s)}{\mathrm{d} s}\right|_{s=0}=\int \cdots \int_{[0,1]^{N}} \log \left|P\left(e^{2 \pi i \theta_{1}}, \ldots, e^{2 \pi i \theta_{N}}\right)\right| \mathrm{d} \theta_{1} \cdots \mathrm{d} \theta_{N} .
$$

A straightforward comparison of the two definitions reveals that

$$
W_{N}(s)=Z\left(x_{1}+\cdots+x_{N} ; s\right)=Z\left(1+x_{1}+\cdots+x_{N-1} ; s\right)
$$

and

$$
W_{N}^{\prime}(0)=\mathrm{m}\left(x_{1}+\cdots+x_{N}\right)=\mathrm{m}\left(1+x_{1}+\cdots+x_{N-1}\right)=\int_{0}^{N} p_{N}(x) \log x \mathrm{~d} x,
$$


where the derivative is with respect to $s$. The latter Mahler measures are known as linear Mahler measures. The evaluations

$W_{2}^{\prime}(0)=0, \quad W_{3}^{\prime}(0)=L^{\prime}\left(\chi_{-3} ;-1\right)=\frac{3 \sqrt{3}}{4 \pi} L\left(\chi_{-3} ; 2\right), \quad W_{4}^{\prime}(0)=-14 \zeta^{\prime}(-2)=\frac{7 \zeta(3)}{2 \pi^{2}}$,

are known [24], while the following conjectural evaluations, due to Rodriguez-Villegas [13] and verified to several hundred digits [5], remain open:

$$
\begin{aligned}
& W_{5}^{\prime}(0) \stackrel{?}{=}-L^{\prime}\left(f_{3} ;-1\right)=6\left(\frac{\sqrt{15}}{2 \pi}\right)^{5} L\left(f_{3} ; 4\right), \\
& W_{6}^{\prime}(0) \stackrel{?}{=}-8 L^{\prime}\left(f_{4} ;-1\right)=3\left(\frac{\sqrt{6}}{\pi}\right)^{6} L\left(f_{4} ; 5\right),
\end{aligned}
$$

where

$$
f_{3}(\tau)=\eta(\tau)^{3} \eta(15 \tau)^{3}+\eta(3 \tau)^{3} \eta(5 \tau)^{3} \quad \text { and } \quad f_{4}(\tau)=\eta(\tau)^{2} \eta(2 \tau)^{2} \eta(3 \tau)^{2} \eta(6 \tau)^{2}
$$

are cusp eigenforms of weight 3 and 4, respectively. Here and in what follows, Dedekind's eta function

$$
\eta(\tau)=q^{1 / 24} \prod_{m=1}^{\infty}\left(1-q^{m}\right)=\sum_{n=-\infty}^{\infty}(-1)^{n} q^{(6 n+1)^{2} / 24}, \quad \text { where } q=e^{2 \pi i \tau},
$$

serves as a principal constructor of modular forms and functions. No similar formulae are known for $W_{N}^{\prime}(0)$ when $N \geq 7$, though the story continues at a different level see [14, 30, 31] for details.

\section{Generic tWo-Step RAndom WALKS}

Let $X_{1}$ and $X_{2}$ be two (sufficiently nice, independent) random variables on $[0, \infty$ ) with probability density $p_{1}(x)$ and $p_{2}(x)$, respectively, and let $\theta_{1}$ and $\theta_{2}$ be uniformly distributed on $[0,1]$. Then $X=e^{2 \pi i \theta_{1}} X_{1}+e^{2 \pi i \theta_{2}} X_{2}$ describes a two-step random walk in the plane with a first step of length $X_{1}$ and a second step of length $X_{2}$. As in [10, eq. (3-3)], an application of the cosine rule shows that the $s$-th moment of $|X|$ is

$$
W(s)=\mathrm{E}\left(|X|^{s}\right)=\int_{0}^{\infty} \int_{0}^{\infty} g_{s}(x, y) p_{1}(x) p_{2}(y) \mathrm{d} x \mathrm{~d} y
$$

where

$$
g_{s}(x, y)=\frac{1}{\pi} \int_{0}^{\pi}\left(x^{2}+y^{2}+2 x y \cos \theta\right)^{s / 2} \mathrm{~d} \theta .
$$

Observe that

$$
\left.\frac{\mathrm{d} g_{s}(x, y)}{\mathrm{d} s}\right|_{s=0}=\frac{1}{\pi} \int_{0}^{\pi} \log \sqrt{x^{2}+y^{2}+2 x y \cos \theta} \mathrm{d} \theta=\max \{\log |x|, \log |y|\},
$$

so that, in particular,

Lemma 1. We have

$$
W^{\prime}(0)=\mathrm{E}(\log |X|)=\int_{0}^{\infty} \int_{0}^{\infty} p_{1}(x) p_{2}(y) \max \{\log x, \log y\} \mathrm{d} y \mathrm{~d} x .
$$


Alternative equivalent expressions, that will be useful in what follows, include

$$
\begin{aligned}
\mathrm{E}(\log |X|) & =\int_{0}^{\infty} \int_{0}^{x} p_{1}(x) p_{2}(y) \log x \mathrm{~d} y \mathrm{~d} x+\int_{0}^{\infty} \int_{x}^{\infty} p_{1}(x) p_{2}(y) \log y \mathrm{~d} y \mathrm{~d} x \\
& =\mathrm{E}\left(\log X_{1}\right)+\int_{0}^{\infty} \int_{x}^{\infty} p_{1}(x) p_{2}(y)(\log y-\log x) \mathrm{d} y \mathrm{~d} x \\
& =\mathrm{E}\left(\log X_{2}\right)+\int_{0}^{\infty} \int_{0}^{x} p_{1}(x) p_{2}(y)(\log x-\log y) \mathrm{d} y \mathrm{~d} x
\end{aligned}
$$

\section{Linear Mahler measures}

Let $N, M$ be integers such that $N>M>0$. By decomposing an $N$-step random walk into two walks with $N-M$ and $M$ steps, and applying Lemma 1 in the form (21), we find that

$$
W_{N}^{\prime}(0)=W_{M}^{\prime}(0)+\int_{0}^{N-M} p_{N-M}(x)\left(\int_{0}^{x} p_{M}(y)(\log x-\log y) \mathrm{d} y\right) \mathrm{d} x .
$$

This formula, together with known formulae for the densities [11, like $p_{1}(x)=$ $\delta(x-1)$ (the Dirac delta function) and $p_{2}(x)=2 /\left(\pi \sqrt{4-x^{2}}\right)$ for $0<x<2$, allows one to produce new expressions for linear Mahler measures. Indeed, taking $M=1$ we get

$$
W_{N}^{\prime}(0)=\int_{1}^{N-1} p_{N-1}(x) \log x \mathrm{~d} x
$$

(which can be also derived using Jensen's formula), while $M=2$ results in

$$
W_{N}^{\prime}(0)=\int_{2}^{N-2} p_{N-2}(x) \log x \mathrm{~d} x+\frac{1}{\pi} \int_{0}^{2} p_{N-2}(x) x \cdot{ }_{3} F_{2}\left(\begin{array}{c}
\frac{1}{2}, \frac{1}{2}, \frac{1}{2} \\
\frac{3}{2}, \frac{3}{2}
\end{array} \mid \frac{x^{2}}{4}\right) \mathrm{d} x
$$

(see also [20, eq. (2.1)]). Here, and in what follows, the hypergeometric notation

$$
{ }_{m} F_{m-1}\left(\begin{array}{c}
a_{1}, a_{2}, \ldots, a_{m} \\
b_{2}, \ldots, b_{m}
\end{array} \mid z\right)=\sum_{n=0}^{\infty} \frac{\left(a_{1}\right)_{n}\left(a_{2}\right)_{n} \cdots\left(a_{m}\right)_{n}}{\left(b_{2}\right)_{n} \cdots\left(b_{m}\right)_{n}} \frac{z^{n}}{n !}
$$

is used, where

$$
(a)_{n}=\frac{\Gamma(a+n)}{\Gamma(a)}= \begin{cases}a(a+1) \cdots(a+n-1), & \text { for } n \geq 1, \\ 1, & \text { for } n=0\end{cases}
$$

denotes the Pochhammer symbol (the rising factorial). Note that we deduce (4) from

$$
\int_{0}^{x} p_{2}(y)(\log x-\log y) \mathrm{d} y=\frac{x}{\pi} \cdot{ }_{3} F_{2}\left(\begin{array}{c}
\frac{1}{2}, \frac{1}{2}, \frac{1}{2} \\
\frac{3}{2}, \frac{3}{2}
\end{array} \frac{x^{2}}{4}\right)
$$

which is valid if $0 \leq x \leq 2$.

Equations (3) and (44) and the formula

$$
p_{4}(x)=\frac{2 \sqrt{16-x^{2}}}{\pi^{2} x} \operatorname{Re}_{3} F_{2}\left(\begin{array}{c|c}
\frac{1}{2}, \frac{1}{2}, \frac{1}{6}, \frac{\left(16-x^{2}\right)^{3}}{6} & \left.\frac{(16}{108 x^{4}}\right)
\end{array}\right.
$$


obtained in [11, Theorem 4.9], provide the formulae

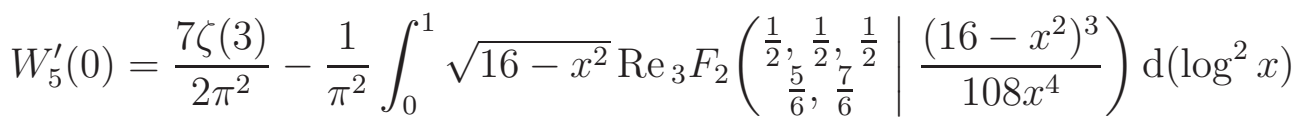

and

$$
\begin{aligned}
& W_{6}^{\prime}(0)=\frac{7 \zeta(3)}{2 \pi^{2}}-\frac{1}{\pi^{2}} \int_{0}^{2} \sqrt{16-x^{2}} \operatorname{Re}_{3} F_{2}\left(\frac{1}{2}, \frac{1}{\frac{5}{6}}, \frac{7}{6}, \frac{1}{2} \mid \frac{\left(16-x^{2}\right)^{3}}{108 x^{4}}\right) \mathrm{d}\left(\log ^{2} x\right) \\
& +\frac{2}{\pi^{3}} \int_{0}^{2} \sqrt{16-x^{2}} \operatorname{Re}_{3} F_{2}\left({ }_{\frac{1}{2}}^{\frac{1}{6}}, \frac{1}{2}, \frac{1}{6} \mid \frac{\frac{1}{2}}{108 x^{4}}\right) \cdot{ }_{3} F_{2}\left(\frac{1}{2}, \frac{1}{2}, \frac{1}{2}, \frac{3}{2} \mid \frac{x^{2}}{4}\right) \mathrm{d} x .
\end{aligned}
$$

These single integrals can be used to numerically confirm the conjectural evaluations of $W_{5}^{\prime}(0)$ and $W_{6}^{\prime}(0)$.

A similar application of Lemma 1, upon decomposing a 6-step walk into two walks with 3 steps, yields the alternative reduction

$$
W_{6}^{\prime}(0)=2 \int_{0}^{3} p_{3}(x) \log x\left(\int_{0}^{x} p_{3}(y) \mathrm{d} y\right) \mathrm{d} x,
$$

where [1]

$$
p_{3}(x)=\frac{2 \sqrt{3} x}{\pi\left(3+x^{2}\right)} \cdot{ }_{2} F_{1}\left(\begin{array}{c|c}
\frac{1}{3}, \frac{2}{3} & \frac{x^{2}\left(9-x^{2}\right)^{2}}{1}
\end{array}\right) .
$$

We discuss this formula further in Section 5 .

Finally, we mention that equation (3) and a modular parametrisation of $p_{4}(x)$ (which we indicate in Section 6) were independently cast in [23] to produce a double $L$-value expression for $W_{5}^{\prime}(0)$.

\section{Modular parametrisation of $p_{3}(x)$ And Related Formulae}

Note that formula (5) can be written as

$$
W_{6}^{\prime}(0)=\int_{0}^{3} \log x \mathrm{~d}\left(P_{3}(x)^{2}\right)=\log 3-\int_{0}^{3} P_{3}(x)^{2} \frac{\mathrm{d} x}{x},
$$

featuring the cumulative density function

$$
P_{3}(x)=\int_{0}^{x} p_{3}(y) \mathrm{d} y .
$$

The related modular parametrisation of $p_{3}(x)$ is given by

$$
x=x(\tau)=3 \frac{\eta(\tau)^{2} \eta(6 \tau)^{4}}{\eta(2 \tau)^{4} \eta(3 \tau)^{2}}:(i \infty, 0) \rightarrow(0,3),
$$

so that

$$
p_{3}(x)=\frac{2 \sqrt{3}}{\pi} \frac{\eta(2 \tau)^{2} \eta(6 \tau)^{2}}{\eta(\tau) \eta(3 \tau)}, \quad \mathrm{d} x=3 \pi i \frac{\eta(\tau)^{6} \eta(3 \tau)^{2} \eta(6 \tau)^{2}}{\eta(2 \tau)^{6}} \mathrm{~d} \tau
$$

and

$$
P_{3}(x)=6 i \sqrt{3} \int_{i \infty}^{\tau} \frac{\eta(\tau)^{5} \eta(3 \tau) \eta(6 \tau)^{4}}{\eta(2 \tau)^{4}} \mathrm{~d} \tau
$$


is the anti-derivative of a weight 3 holomorphic Eisenstein series

$$
\frac{\eta(\tau)^{5} \eta(3 \tau) \eta(6 \tau)^{4}}{\eta(2 \tau)^{4}}=E_{3, \chi_{-3}}(\tau)-8 E_{3, \chi_{-3}}(2 \tau)
$$

where

$$
E_{3, \chi_{-3}}(\tau)=\frac{\eta(3 \tau)^{9}}{\eta(\tau)^{3}}=\sum_{m, n=1}^{\infty}\left(\frac{-3}{m}\right) n^{2} q^{m n}, \quad \chi_{-3}(m)=\left(\frac{-3}{m}\right)=\frac{e^{2 \pi i m / 3}-e^{-2 \pi i m / 3}}{i \sqrt{3}} .
$$

Though the anti-derivative $P_{3}(x)$,

$$
\begin{aligned}
P_{3}(x) & =\frac{3 \sqrt{3}}{\pi}\left(\sum_{m, n=1}^{\infty}\left(\frac{-3}{m}\right) \frac{n}{m} q^{m n}-4 \sum_{m, n=1}^{\infty}\left(\frac{-3}{m}\right) \frac{n}{m} q^{2 m n}\right) \\
& =\frac{9 i}{\pi} \log \prod_{n=1}^{\infty}\left(\frac{\left(1-e^{2 \pi i / 3} q^{2 n}\right)^{4}\left(1-e^{-2 \pi i / 3} q^{n}\right)}{\left(1-e^{-2 \pi i / 3} q^{2 n}\right)^{4}\left(1-e^{2 \pi i / 3} q^{n}\right)}\right)^{n},
\end{aligned}
$$

is not considered to be sufficiently "natural", it shows up as the elliptic dilogarithm thanks to Bloch's formula; see [17, 19] for the details. Note that

$$
E_{3, \chi_{-3}}\left(-\frac{1}{3 \tau}\right)=\frac{i \tau^{3}}{3 \sqrt{3}} \tilde{E}_{3, \chi_{-3}}(\tau), \quad \tilde{E}_{3, \chi_{-3}}(\tau)=\frac{\eta(\tau)^{9}}{\eta(3 \tau)^{3}}=1-9 \sum_{m, n=1}^{\infty}\left(\frac{-3}{n}\right) n^{2} q^{m n}
$$

and, in addition, we have

$$
\begin{aligned}
\frac{1}{2 \pi i} \frac{\mathrm{d} x / \mathrm{d} \tau}{x}=\frac{1}{2}\left(\frac{\eta(\tau)^{2} \eta(3 \tau)^{2}}{\eta(2 \tau) \eta(6 \tau)}\right)^{2} & =\frac{1}{18}\left(E_{1, \chi_{-3}}(\tau)-4 E_{1, \chi_{-3}}(4 \tau)\right)^{2} \\
& =\frac{1}{54 \tau^{2}}\left(E_{1, \chi_{-3}}\left(-\frac{1}{12 \tau}\right)-E_{1, \chi_{-3}}\left(-\frac{1}{3 \tau}\right)\right)^{2},
\end{aligned}
$$

where

$$
E_{1, \chi-3}(\tau)=1+6 \sum_{m, n=1}^{\infty}\left(\frac{-3}{m}\right) q^{m n}
$$

\section{Modular computation for $W_{5}^{\prime}(0)$ And $W_{6}^{\prime}(0)$}

As (partly) shown in [11] the density $p_{4}(x)$ can be parameterised as follows (we make a shift of $\tau$ by half):

$$
p_{4}(x(\tau))=-\operatorname{Re}\left(\frac{2 i\left(1+6 \tau+12 \tau^{2}\right)}{\pi} p(\tau)\right)
$$

where

$$
p(\tau)=\frac{\eta(2 \tau)^{4} \eta(6 \tau)^{4}}{\eta(\tau) \eta(3 \tau) \eta(4 \tau) \eta(12 \tau)} \quad \text { and } \quad x(\tau)=\left(\frac{2 \eta(\tau) \eta(3 \tau) \eta(4 \tau) \eta(12 \tau)}{\eta(2 \tau)^{2} \eta(6 \tau)^{2}}\right)^{3}
$$

The path for $\tau$ along the imaginary axis from 0 to $i /(2 \sqrt{3})$ (or from $i \infty$ to $i /(2 \sqrt{3})$ ) corresponds to $x$ ranging from 0 to 2 , while the path from $i /(2 \sqrt{3})$ to $-1 / 4+i /(4 \sqrt{3})$ along the arc centred at 0 corresponds to the real range $(2,4)$ for $x$. (The arc admits 
the parametrisation $\tau=e^{\pi i \theta} /(2 \sqrt{3}), 1 / 2<\theta<5 / 6$. $)$ Note that $x(i /(2 \sqrt{15}))=1$ and

$$
p_{4}(x(\tau))= \begin{cases}-\frac{2 i \cdot 6 \tau}{\pi} p(\tau), & \text { for } \tau \text { on the imaginary axis } \\ -\frac{2 i\left(1+6 \tau+12 \tau^{2}\right)}{\pi} p(\tau), & \text { for } \tau \text { on the arc }\end{cases}
$$

and

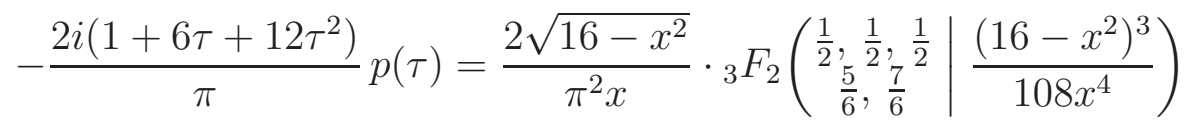

(this is a general form of [11, Theorem 4.9]). Formulas (11), (3) and (41) reduce the conjectural evaluations of $W_{5}^{\prime}(0)$ and $W_{6}^{\prime}(0)$ to the following ones:

$$
\frac{7 \zeta(3)}{2 \pi^{2}}+L^{\prime}\left(f_{3} ;-1\right) \stackrel{?}{=} \frac{12}{\pi} \int_{0}^{1 /(2 \sqrt{15})} y p(i y) \log x(i y) \mathrm{d} x(i y)
$$

and

$$
\begin{aligned}
\frac{7 \zeta(3)}{2 \pi^{2}}+8 L^{\prime}\left(f_{4} ;-1\right) & \stackrel{?}{=} \frac{12}{\pi} \int_{0}^{1 /(2 \sqrt{3})} y p(i y) \log x(i y) \mathrm{d} x(i y) \\
- & \frac{12}{\pi^{2}} \int_{0}^{1 /(2 \sqrt{3})} y p(i y) x(i y) \cdot{ }_{3} F_{2}\left(\frac{1}{2}, \frac{1}{2}, \frac{3}{2}, \frac{1}{2} \mid \frac{x(i y)^{2}}{4}\right) \mathrm{d} x(i y) .
\end{aligned}
$$

Furthermore, note that the Atkin-Lehner involutions $w_{12}: \tau \mapsto-1 /(12 \tau)$ and $w_{6}: \tau \mapsto(6 \tau-5) /(12 \tau-6)$ act on the modular function $x(\tau)$ as follows: $x\left(w_{12} \tau\right)=$ $x(\tau)$ and $x\left(w_{6} \tau\right)=-8 / x(\tau)$, and we also have $p\left(w_{12} \tau\right)=-\tau^{2} p(\tau)$. The point $i /(2 \sqrt{3})$ is fixed by $w_{12}$. Thus, the change of variable $y \mapsto 1 /(12 y)$ leads to

$$
\int_{0}^{1 /(2 \sqrt{3})} y p(i y) \log x(i y) \mathrm{d} x(i y)=-\int_{1 /(2 \sqrt{3})}^{\infty} y p(i y) \log x(i y) \mathrm{d} x(i y) .
$$

\section{Mahler measures Related to A VARiation of RANDOM WALK}

In [23] the Mahler measures $\mathrm{m}\left(1+x_{1}+x_{2}\right)$ and $\mathrm{m}\left(1+x_{1}+x_{2}+x_{3}\right)$ are computed using the modular parametrisations of

$$
\sum_{n=0}^{\infty} W_{3}(2 n) z^{n}=\sum_{n=0}^{\infty} \mathrm{CT}\left(\left(1+x_{1}+x_{2}\right)\left(1+x_{1}^{-1}+x_{2}^{-1}\right)\right)^{n} z^{n}
$$

and

$$
\sum_{n=0}^{\infty} W_{4}(2 n) z^{n}=\sum_{n=0}^{\infty} \mathrm{CT}\left(\left(1+x_{1}+x_{2}+x_{3}\right)\left(1+x_{1}^{-1}+x_{2}^{-1}+x_{3}^{-1}\right)\right)^{n} z^{n},
$$

where $\mathrm{CT}(L)$ denotes the constant term of a Laurent polynomial $L \in \mathbb{Z}\left[x_{1}^{ \pm}, x_{2}^{ \pm}, \ldots\right]$. Note that the Picard-Fuchs linear differential equations for the two generating functions give rise to the ones for the densities $p_{3}(x)$ and $p_{4}(x)$ together with their explicit hypergeometric and modular expressions (see [11, eq. (3.2) and Remark 4.10]), though it remains unclear whether the latter information can be used to compute $W_{N}^{\prime}(0)$ in (1) for $N=3,4$. This is itself an interesting question to not only assist in 
computing of $W_{N}^{\prime}(0)$ for $N>4$ but also in relation with another famous conjecture of Boyd:

$$
\mathrm{m}\left(1+x_{1}+x_{2}+x_{3}+x_{2} x_{3}\right) \stackrel{?}{=}-2 L^{\prime}\left(f_{2} ;-1\right)=\frac{15^{2}}{4 \pi^{4}} L\left(f_{2} ; 3\right)=0.4839979734 \ldots
$$

where $f_{2}(\tau)=\eta(\tau) \eta(3 \tau) \eta(5 \tau) \eta(15 \tau)$.

In analogy with the case of linear Mahler measures, we define

$$
\begin{aligned}
\widetilde{W}(s) & =\iiint_{[0,1]^{3}}\left|1+e^{2 \pi i \theta_{1}}+e^{2 \pi i \theta_{2}}+e^{2 \pi i \theta_{3}}+e^{2 \pi i\left(\theta_{2}+\theta_{3}\right)}\right|^{s} \mathrm{~d} \theta_{1} \mathrm{~d} \theta_{2} \mathrm{~d} \theta_{3} \\
& =Z\left(1+x_{1}+x_{2}+x_{3}+x_{2} x_{3} ; s\right)
\end{aligned}
$$

as the $s$-th moment of a random 5-step walk for which the direction of the final step is completely determined by the two previous steps. Then the even moments

$$
\begin{aligned}
\widetilde{W}(2 n) & =\mathrm{CT}\left(\left(1+x_{1}+x_{2}+x_{3}+x_{2} x_{3}\right)\left(1+x_{1}^{-1}+x_{2}^{-1}+x_{3}^{-1}+\left(x_{2} x_{3}\right)^{-1}\right)\right)^{n} \\
& =\sum_{k=0}^{n}\left(\begin{array}{l}
n \\
k
\end{array}\right)^{2}\left(\begin{array}{c}
2 k \\
k
\end{array}\right)^{2}
\end{aligned}
$$

satisfy a rather lengthy recurrence equation, which is equivalent to a Picard-Fuchs differential equation of order 4 . The latter splits into the tensor product of two differential equations of order 2 and, with some effort, we obtain the following result.

Theorem 1. We have

$$
\begin{aligned}
& \sum_{n=0}^{\infty} \widetilde{W}(2 n)\left(\frac{t}{(4+t)(1+4 t)}\right)^{n} \\
& \quad=\frac{(4+t)(1+4 t)}{4\left(1+4 t+t^{2}\right)}{ }_{2} F_{1}\left(\begin{array}{c|c}
\frac{1}{2}, & \frac{1}{2} \\
1 & \left.\frac{t(4+t)}{1+4 t+t^{2}}\right) \cdot{ }_{2} F_{1}\left(\begin{array}{c}
\frac{1}{2}, \\
1
\end{array} \mid \frac{t^{2}}{1+4 t+t^{2}}\right)
\end{array}\right.
\end{aligned}
$$

and, more generally,

$$
\begin{aligned}
& \frac{b}{(b+t)(1+b t)} \sum_{n=0}^{\infty}\left(\frac{t}{(b+t)(1+b t)}\right)^{n} \sum_{k=0}^{n}\left(\begin{array}{l}
n \\
k
\end{array}\right)^{2}\left(\begin{array}{c}
2 k \\
k
\end{array}\right)^{2}\left(\frac{b}{4}\right)^{2 k}
\end{aligned}
$$

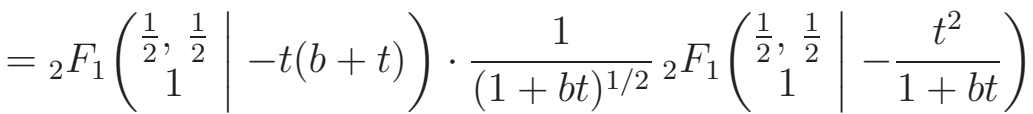

$$
\begin{aligned}
& =\frac{1}{1+b t+t^{2}}{ }_{2} F_{1}\left(\begin{array}{c|c}
\frac{1}{2}, \frac{1}{2} & \frac{t(b+t)}{1}
\end{array}\right) \cdot{ }_{2} F_{1}\left(\begin{array}{c|c}
\frac{1}{2}, \frac{1}{2} & \frac{t^{2}}{1+b t+t^{2}}
\end{array}\right) \text {. }
\end{aligned}
$$

Proof. Once a factorisation of this type is written down, it is a computational routine to prove it. In other words, a principal issue is discovering such a formula rather than proving it. Our original discovery of Theorem 1 involved a lot of experimental mathematics; however, we later realised that it is deducible from known formulae 
as follows:

$$
\begin{aligned}
& \sum_{n=0}^{\infty} z^{n} \sum_{k=0}^{n}\left(\begin{array}{l}
n \\
k
\end{array}\right)^{2}\left(\begin{array}{c}
2 k \\
k
\end{array}\right)^{2} x^{k}=\sum_{k=0}^{\infty}\left(\begin{array}{c}
2 k \\
k
\end{array}\right)^{2} x^{k} \sum_{m=0}^{\infty}\left(\begin{array}{c}
k+m \\
k
\end{array}\right)^{2} z^{k+m} \\
& =\sum_{k=0}^{\infty}\left(\begin{array}{c}
2 k \\
k
\end{array}\right)^{2}(x z)_{2}^{k} F_{1}\left(\begin{array}{c|c}
k+1, k+1 & z \\
1 & z
\end{array}\right) \\
& =\sum_{k=0}^{\infty}\left(\begin{array}{c}
2 k \\
k
\end{array}\right)^{2} \frac{(x z)^{k}}{(1-z)^{k+1}}{ }_{2} F_{1}\left(\begin{array}{c|c}
-k, k+1 & -\frac{z}{1-z} \\
1 & -2
\end{array}\right. \\
& =\frac{1}{1-z} \sum_{k=0}^{\infty}\left(\begin{array}{c}
2 k \\
k
\end{array}\right)^{2}\left(\frac{x z}{1-z}\right)^{k} \cdot P_{k}\left(\frac{1+z}{1-z}\right) \text {, }
\end{aligned}
$$

where $P_{k}$ denotes the $k$-th Legendre polynomial, and the latter generating function is a particular instance of the Bailey-Brafman formula [15, 34].

We remark that, using the general Bailey-Brafman formula and its generalisation from [29], the proof above extends to the factorisation of the two-variable generating functions

$$
\sum_{n=0}^{\infty} z^{n} \sum_{k=0}^{n}\left(\begin{array}{l}
n \\
k
\end{array}\right)^{2} \frac{(s)_{k}(1-s)_{k}}{k !^{2}} x^{k}
$$

as well as of

$$
\sum_{n=0}^{\infty} z^{n} \sum_{k}\left(\begin{array}{c}
n \\
2 k
\end{array}\right)^{2}\left(\begin{array}{c}
2 k \\
k
\end{array}\right)^{2} x^{k} \text { and } \sum_{n=0}^{\infty} z^{n} \sum_{k}\left(\begin{array}{c}
n \\
3 k
\end{array}\right)^{2} \frac{(3 k) !}{k !^{3}} x^{k}
$$

and even of

$$
\sum_{n=0}^{\infty} z^{n} \sum_{k=0}^{n}\left(\begin{array}{l}
n \\
k
\end{array}\right)^{2} u_{k} x^{k}
$$

for an Apéry-like sequence $u_{0}, u_{1}, u_{2}, \ldots$.

Furthermore, we expect that Theorem 1 can lead to a hypergeometric expression for the density function $\widetilde{p}(x)$ (piecewise analytic, with finite support on the interval $0<x<5)$, which is the inverse Mellin transform of $\widetilde{W}(s-1)$, hence to the Mahler measure evaluation

$$
\mathrm{m}\left(1+x_{1}+x_{2}+x_{3}+x_{2} x_{3}\right)=\widetilde{W}^{\prime}(0)=\int_{0}^{\infty} \widetilde{p}(x) \log x \mathrm{~d} x=\int_{0}^{5} \widetilde{p}(x) \log x \mathrm{~d} x .
$$

On the other hand, the reduction technique of Sections 3 and 4 suggests a different approach to computing $\widetilde{W^{\prime}}(0)$, resulting in the following hypergeometric evaluation of the Mahler measure.

Theorem 2. We have

$$
\mathrm{m}\left(1+x_{1}+x_{2}+x_{3}+x_{2} x_{3}\right)=-\frac{1}{2 \pi} \int_{0}^{1}{ }_{2} F_{1}\left(\begin{array}{c}
\frac{1}{2}, \frac{1}{2} \\
1
\end{array} \mid 1-\frac{x^{2}}{16}\right) \log x \mathrm{~d} x .
$$


Proof. Define a related density $\widehat{p}(x)$ by

$$
\begin{aligned}
\int_{0}^{4} x^{s} \widehat{p}(x) \mathrm{d} x & =\widehat{W}(s)=\iint_{[0,1]^{2}}\left|1+e^{2 \pi i \theta_{2}}+e^{2 \pi i \theta_{3}}+e^{2 \pi i\left(\theta_{2}+\theta_{3}\right)}\right|^{s} \mathrm{~d} \theta_{2} \mathrm{~d} \theta_{3} \\
& =W_{2}(s)^{2}=\frac{\Gamma(1+s)^{2}}{\Gamma(1+s / 2)^{4}}
\end{aligned}
$$

By an application of the Mellin transform calculus, we find that, for $0<x<4$,

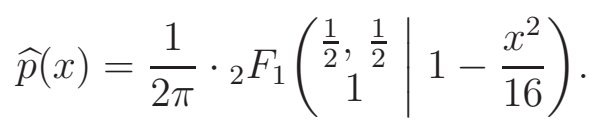

Then it follows from Lemma 1 that

$$
\widetilde{W^{\prime}}(0)=\int_{1}^{4} \widehat{p}(x) \log x \mathrm{~d} x=-\int_{0}^{1} \widehat{p}(x) \log x \mathrm{~d} x,
$$

where we use the evaluation

$$
\int_{0}^{4} \widehat{p}(x) \log x \mathrm{~d} x=\mathrm{m}\left(1+x_{2}+x_{3}+x_{2} x_{3}\right)=\mathrm{m}\left(1+x_{2}\right)+\mathrm{m}\left(1+x_{3}\right)=0 .
$$

The above proof extends to the general formula

$$
\begin{aligned}
\mathrm{m}\left(1+b x_{1}+x_{2}+x_{3}+x_{2} x_{3}\right) & =\log b \int_{0}^{b} \widehat{p}(x) \mathrm{d} x+\int_{b}^{4} \widehat{p}(x) \log x \mathrm{~d} x \\
& =\frac{1}{2 \pi} \int_{0}^{b}{ }_{2} F_{1}\left(\begin{array}{c}
\frac{1}{2}, \frac{1}{2} \\
1
\end{array} 1-\frac{x^{2}}{16}\right) \log \frac{b}{x} \mathrm{~d} x
\end{aligned}
$$

for $0<b \leq 4$. A related computation

$$
\mathrm{m}\left(1+b x_{1}+x_{2}+x_{3}+x_{2} x_{3}\right)=\log b+\frac{8}{\pi^{2}} \int_{b}^{4} \frac{\arccos (b / x) \log (x /(2 \sqrt{b}))}{\sqrt{16-x^{2}}} \mathrm{~d} x
$$

valid for $0<b \leq 4$ was given by J. Wan [27]; he also pointed out that $\mathrm{m}\left(1+b x_{1}+\right.$ $\left.x_{2}+x_{3}+x_{2} x_{3}\right)=\log b$ for $b>4$ follows from Jensen's formula.

The left-hand side of another Mahler measure conjecture [13]

$$
\mathrm{m}\left(\left(1+x_{1}\right)^{2}+x_{2}+x_{3}\right) \stackrel{?}{=}-L^{\prime}\left(\tilde{f}_{2} ;-1\right)=\frac{72}{\pi^{4}} L\left(\tilde{f}_{2} ; 3\right)=0.7025655062 \ldots,
$$

where $\tilde{f}_{2}(\tau)=\eta(2 \tau) \eta(4 \tau) \eta(6 \tau) \eta(12 \tau)$ is a cusp form of level 24 , can be treated by a similar reduction, using that the densities for $\left(1+x_{1}\right)^{2}$ and $x_{2}+x_{3}$ are $p_{2}\left(t^{1 / 2}\right) /\left(2 t^{1 / 2}\right)$ on $[0,4]$ and $p_{2}(t)$ on $[0,2]$, respectively. The final result is the elegant formula

$$
\mathrm{m}\left(\left(1+x_{1}\right)^{2}+x_{2}+x_{3}\right)=\frac{2 G}{\pi}+\frac{2}{\pi^{2}} \int_{0}^{1} \arcsin (1-x) \arcsin x \frac{\mathrm{d} x}{x},
$$

where $G$ is Catalan's constant, and, with some further work, we can express the right-hand side hypergeometrically. 
Theorem 3. We have

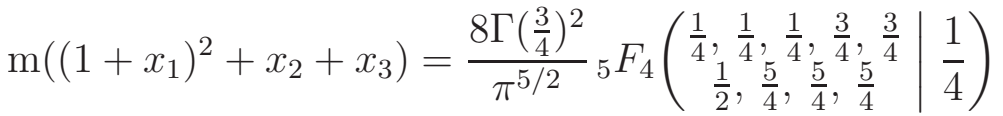

$$
\begin{aligned}
& +\frac{\Gamma\left(\frac{1}{4}\right)^{2}}{54 \pi^{5 / 2}}{ }_{5} F_{4}\left(\begin{array}{c}
\frac{3}{4}, \frac{3}{4}, \frac{3}{4}, \frac{5}{4}, \frac{5}{4}, \frac{1}{4}, \frac{7}{4}, \frac{7}{4} \\
\frac{3}{4}
\end{array}\right) .
\end{aligned}
$$

Proof. Notice that, for $0<x<1$,

$$
\arcsin (1-x)=\frac{\pi}{2}-\arccos (1-x)=\frac{\pi}{2}-\sqrt{2 x}_{2} F_{1}\left(\begin{array}{c|c}
\frac{1}{2}, \frac{1}{2} & \frac{x}{2} \\
\frac{3}{2}
\end{array}\right),
$$

and that, for $n>-1 / 2$,

$$
\int_{0}^{1} x^{n-1 / 2} \arcsin x \mathrm{~d} x=\frac{\sqrt{\pi}}{2 n+1}\left(\sqrt{\pi}-\frac{\Gamma\left(\frac{n}{2}+\frac{3}{4}\right)}{\Gamma\left(\frac{n}{2}+\frac{5}{4}\right)}\right) .
$$

Therefore,

$$
\begin{aligned}
\int_{0}^{1} \arcsin (1-x) \arcsin x \frac{\mathrm{d} x}{x}=\frac{\pi}{2} & \int_{0}^{1} \arcsin x \frac{\mathrm{d} x}{x}-\pi \sqrt{2} \sum_{n=0}^{\infty} \frac{\left(\frac{1}{2}\right)_{n}^{2}}{n !\left(\frac{3}{2}\right)_{n}(2 n+1)} \frac{1}{2^{n}} \\
& +\sqrt{2 \pi} \sum_{n=0}^{\infty} \frac{\left(\frac{1}{2}\right)_{n}^{2} \Gamma\left(\frac{n}{2}+\frac{3}{4}\right)}{n !\left(\frac{3}{2}\right)_{n}(2 n+1) \Gamma\left(\frac{n}{2}+\frac{5}{4}\right)} \frac{1}{2^{n}} .
\end{aligned}
$$

From this and (17) we deduce

$$
\begin{aligned}
& \mathrm{m}\left(\left(1+x_{1}\right)^{2}+x_{2}+x_{3}\right)=\frac{2 G}{\pi}+\frac{\log 2}{2}-\frac{2 \sqrt{2}}{\pi}{ }_{3} F_{2}\left(\begin{array}{c}
\frac{1}{2}, \frac{1}{2}, \frac{1}{2} \\
\frac{3}{2}, \frac{3}{2}
\end{array}\right.
\end{aligned}
$$

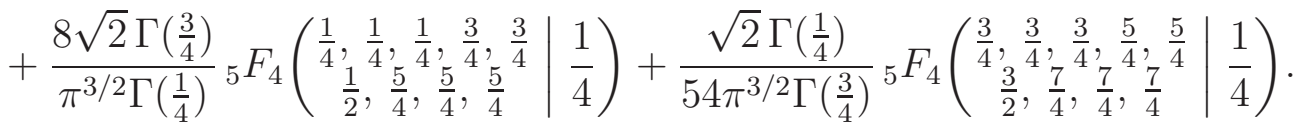

It remains to use

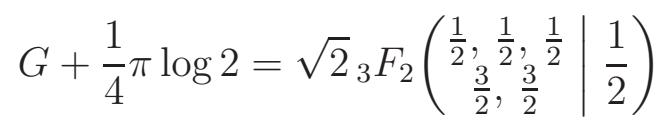

(see [1, Entry 30]) and $\Gamma\left(\frac{1}{4}\right) \Gamma\left(\frac{3}{4}\right)=\pi \sqrt{2}$.

\section{Conclusion}

A goal of this final section is to highlight relevance for and links with other research and open problems.

The (hypergeometric) factorisation in Theorem 1 and similar results outlined after its proof are part of a general phenomenon of arithmetic differential equations of order 4. These are the first instances "beyond modularity" in the sense that arithmetic differential equations of order 2 and 3 are always supplied by modular parametrisation. In order 4 , we have to distinguish two particular novel situations (though our knowledge about either is imperfect and incomplete): (the Zariski closure of) the monodromy group is the orthogonal group $O_{4} \simeq O_{2,2}$ of dimension 6 or the symplectic group $S p_{4}$ of dimension 10. The example given in Theorem 1 corresponds to the first (orthogonal) situation: on the level of Lie groups, $\mathrm{O}_{2,2}$ can 
be realised as the tensor product of two copies of $S L_{2}$ (or $G L_{2}$ ). There is a limited amount of further examples of this type [21, 29, 33] though we expect that all underlying Picard-Fuchs differential equations with such monodromy can be represented as tensor products of two arithmetic differential equations of order 2. There is a natural hypergeometric production of such orthogonal cases using Orr-type formulae (see [18, 28]) but there are plenty of other cases coming from classical work of W. N. Bailey and its recent generalisations [29, 34]. Many such cases, mostly forecast by Sun [25], are still awaiting their explicit factorisation. Though these situations do not cover symplectic monodromy instances, they can still be viewed as an intermediate step between classical modularity and $S p_{4}$ : the antisymmetric square of the latter happens to be $O_{5} \simeq O_{3,2}$ (see [4]).

More in the direction of three-variable Mahler measure, the conjectural evaluation in (66) and Theorem 2 brings us to the expectation

$$
\frac{1}{2 \pi} \int_{0}^{1}{ }_{2} F_{1}\left(\frac{1}{2}, \frac{1}{2} \mid 1-\frac{x^{2}}{16}\right) \log x \mathrm{~d} x \stackrel{?}{=} 2 L^{\prime}\left(f_{2} ;-1\right) .
$$

This one highly resembles the evaluation

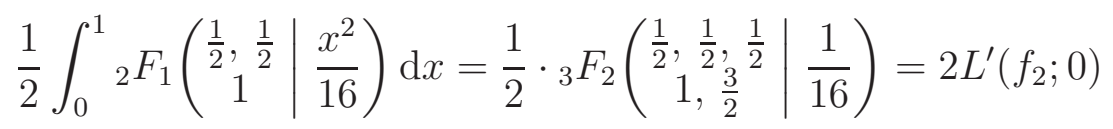

established in [22]. The related modular parametrisation

$$
x=x(\tau)=16\left(\frac{\eta(\tau) \eta(4 \tau)^{2}}{\eta(2 \tau)^{3}}\right)^{4}
$$

corresponds to

$$
\begin{gathered}
1-\frac{x^{2}}{16}=\left(\frac{\eta(\tau)^{2} \eta(4 \tau)}{\eta(2 \tau)^{3}}\right)^{8}, \\
F\left(\frac{x^{2}}{16}\right)=\frac{\eta(2 \tau)^{10}}{\eta(\tau)^{4} \eta(4 \tau)^{4}} \text { and } F\left(1-\frac{x^{2}}{16}\right)=-2 i \tau F\left(\frac{x^{2}}{16}\right),
\end{gathered}
$$

where $F$ denotes the corresponding ${ }_{2} F_{1}$ hypergeometric series. Note that $x$ ranges from 0 to 4 when $\tau$ runs from $i \infty$ to 0 along the imaginary axis; however, the point $\tau_{0}=i 0.8774376613482 \ldots$, at which $x\left(\tau_{0}\right)=1$, is not a quadratic irrationality. Furthermore, H. Cohen [16] observes another step in the ladder (9), (8):

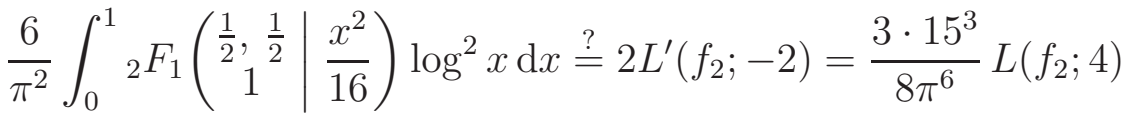

$$
\begin{aligned}
& =1.2165632526 \ldots \text {, }
\end{aligned}
$$

though not linked to a particular Mahler measure. 
The expression in Theorem 3 is somewhat different from the one in Theorem 2 , and resembles the hypergeometric evaluation of the $L$-value

$$
\begin{aligned}
-L^{\prime}\left(\hat{f}_{2} ;-1\right)= & \frac{128}{\pi^{4}} L\left(\hat{f}_{2} ; 3\right) \\
= & \frac{\Gamma\left(\frac{1}{4}\right)^{2}}{6 \sqrt{2} \pi^{5 / 2}}{ }_{4} F_{3}\left(\begin{array}{c}
1,1,1, \frac{1}{2} \mid \\
\frac{7}{4}, \frac{3}{2}, \frac{3}{2} \mid
\end{array}\right)+\frac{4 \Gamma\left(\frac{3}{4}\right)^{2}}{\sqrt{2} \pi^{5 / 2}}{ }_{4} F_{3}\left(\begin{array}{c}
1,1,1, \frac{1}{2} \\
\frac{5}{4}, \frac{3}{2}, \frac{3}{2}
\end{array}\right) \\
& \quad+\frac{\Gamma\left(\frac{1}{4}\right)^{2}}{2 \sqrt{2} \pi^{5 / 2}}{ }_{4} F_{3}\left(\begin{array}{c}
1,1,1, \frac{1}{2} \\
\frac{3}{4}, \frac{3}{2}, \frac{3}{2}
\end{array}\right),
\end{aligned}
$$

where $\hat{f}_{2}(\tau)=\eta(4 \tau)^{2} \eta(8 \tau)^{2}$ is a cusp form of level 32, obtained in [32, Theorem 3].

Finally, we remark that the integral

$$
W_{3}^{\prime}(0)=\int_{0}^{3} \log x \mathrm{~d} P_{3}(x)=\log 3-\int_{0}^{3} P_{3}(x) \frac{\mathrm{d} x}{x}
$$

in the notation of Section 5, with $P_{3}(x)$ related to eta quotients, is visually linked to the following result in [7] (also discussed in greater generality in [2, 26])

$$
\int_{0}^{1} \frac{1}{9}\left(1-\frac{\eta(\tau)^{9}}{\eta(3 \tau)^{3}}\right) \frac{\mathrm{d} q}{q}=\lim _{q \rightarrow 1^{-}} \sum_{m, n=1}^{\infty}\left(\frac{-3}{n}\right) \frac{n}{m} q^{m n}=L^{\prime}\left(\chi_{-3} ;-1\right) .
$$

However, apart from the fact that the two quantities coincide we could not find a direct link between the two integrals.

Acknowledgements. We thank H. Cohen for supplying us with the numerical observation (10) whose origin remains completely mysterious to us. We also thank the referee for their valuable feedback.

\section{REFERENCES}

[1] V.S. AdAmснik, Integral and series representations for Catalan's constant, unpublished.

[2] S. Ahlgren, B. C. Berndt, A. Y. YeE and A. Zaharescu, Integrals of Eisenstein series and derivatives of L-functions, Intern. Math. Res. Not. 2002:32 (2002), 1723-1738.

[3] H. Akatsuka, Zeta Mahler measures, J. Number Theory 129:11 (2009), 2713-2734.

[4] G. Almkvist, D. van Straten and W. Zudilin, Generalizations of Clausen's formula and algebraic transformations of Calabi-Yau differential equations, Proc. Edinburgh Math. Soc. $\mathbf{5 4 : 2}$ (2011), 273-295.

[5] D. H. Bailey and J. M. Borwein, Hand-to-hand combat with multi-thousand-digit integrals, J. Comput. Sci. 3 (2012), 77-86.

[6] D. H. Bailey, J. M. Borwein, D. J. Broadhurst and M. L. Glasser, Elliptic integral evaluations of Bessel moments and applications, J. Phys. A 41:20 (2008), 5203-5231.

[7] B. C. Berndt and A. Zaharescu, An integral of Dedekind eta-functions in Ramanujan's lost notebook, J. Reine Angew. Math. 551 (2002), 33-39.

[8] J. M. Borwein, A short walk can be beautiful, J. Humanistic Math. 6:1 (2016), 86-109.

[9] J. M. Borwein, D. Nuyens, A. Straub and J. Wan, Some arithmetic properties of short random walk integrals, Ramanujan J. 26:1 (2011), 109-132.

[10] J. M. Borwein, A. Straub and J. Wan, Three-step and four-step random walk integrals, Experiment. Math. 22:1 (2013), 1-14.

[11] J. M. Borwein, A. Straub, J. Wan and W. Zudilin, Densities of short uniform random walks, with an appendix by D. Zagier, Canad. J. Math. 64:5 (2012), 961-990. 
[12] D. Boyd, Mahler's measure and special values of L-functions, Experiment. Math. 7:1 (1998), $37-82$.

[13] D. Boyd, D. Lind, F. Rodriguez-Villegas and C. Deninger, The many aspects of Mahler's measure, Final report of the Banff workshop 03w5035 (26 April-1 May 2003).

[14] D. Broadhurst, Feynman integrals, L-series and Kloosterman moments, Comm. Number Theory Phys. 10:3 (2016), 527-569.

[15] H. H. Chan, J. WAN and W. Zudilin, Legendre polynomials and Ramanujan-type series

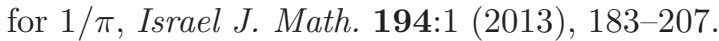

[16] H. Cohen, Personal communication (23 March 2018).

[17] W. Duke and Ö. Imamō̄Lu, On a formula of Bloch, Functiones Approxim. 37.1 (2007), $109-117$.

[18] J. Guillera, A family of Ramanujan-Orr formulas for $1 / \pi$, Integral Transforms Spec. Funct. 26:7 (2015), 531-538.

[19] V. Paşol and W. Zudilin, A study of elliptic gamma function and allies, Res. Math. Sci. (to appear); Preprint arXiv: 1801.00210 [math.NT] (2018), 12 pp.

[20] M. D. Rogers, A study of inverse trigonometric integrals associated with three-variable Mahler measures, and some related identities, J. Number Theory 121 (2006), 265-304.

[21] M. D. Rogers and A. Straub, A solution of Sun's $\$ 520$ challenge concerning 520/ $\pi$, Intern. J. Number Theory 9 (2013), 1273-1288.

[22] M. D. Rogers and W. Zudilin, On the Mahler measure of $1+X+1 / X+Y+1 / Y$, Intern. Math. Research Not. 2014:9 (2014), 2305-2326.

[23] E. Shinder and M. Vlasenko, Linear Mahler measures and double $L$-values of modular forms, J. Number Theory 142 (2014), 149-182.

[24] C.J. Sмyтн, On measures of polynomials in several variables, Bull. Austral. Math. Soc. 23 (1981), 49-63.

[25] Z.-W. Sun, List of conjectural series for powers of $\pi$ and other constants, Preprint arXiv: 1102.5649v47 [math.CA] (December 29, 2014), 33 pp.

[26] R. Takloo-Bighash, A remark on a paper of S. Ahlgren, B. C. Berndt, A. J. Yee, and A. Zaharescu: "Integrals of Eisenstein series and derivatives of $L$-functions" 2, Intern. $J$. Number Theory 2:1 (2006), 111-114.

[27] J. G. Wan, Personal communication (26 July 2011).

[28] J. G. WAN, Series for 1/ $\pi$ using Legendre's relation, Integral Transforms Spec. Funct. 25:1 (2014), 1-14.

[29] J. G. WAn and W. ZudiLin, Generating functions of Legendre polynomials: a tribute to Fred Brafman, J. Approximation Theory 164 (2012), 488-503.

[30] Y. Zhou, Wick rotations, Eichler integrals, and multi-loop Feynman diagrams, Comm. Number Theory Phys.12:1 (2018), 127-192.

[31] Y. ZHOU, On Borwein's conjectures for planar uniform random walks, Preprint arXiv: 1708.02857 [math.CA] (2017), 16 pp.

[32] W. Zudilin, Period(d)ness of L-values, in Number Theory and Related Fields, In memory of Alf van der Poorten, J. M. Borwein et al. (eds.), Springer Proceedings in Math. Stat. 43 (Springer, New York, 2013), 381-395.

[33] W. Zudilin, A generating function of the squares of Legendre polynomials, Bull. Austral. Math. Soc. 89 (2014), 125-131.

[34] W. Zudilin, Hypergeometric heritage of W. N. Bailey. With an appendix: Bailey's letters to F. Dyson, Preprint arXiv: 1611.08806 [math.NT] (2016), 28 pp. 
Department of Mathematics and Statistics, University of South Alabama, 411 University Blvd N, MSPB 325, Mobile, AL 36688, USA

E-mail address: straub@southalabama.edu

Department of Mathematics, IMAPP, Radboud University, PO Box 9010, 6500 GL NiJMEGEn, NETHERLANDS

E-mail address: w.zudilin@math.ru.nl

School of Mathematical and Physical Sciences, The University of Newcastle, Callaghan, NSW 2308, Australia

E-mail address: wadim.zudilin@newcastle.edu.au

Laboratory of Mirror Symmetry and Automorphic Forms, National Research University Higher School of Economics, 6 Usacheva str., 119048 Moscow, Russia E-mail address: wzudilin@gmail.com 